\title{
Supporting Infgormation
}

Elysiapyrones from Elysia diomedea. Do such Metabolites Evidence an EnzymaticallyAssisted Electrocyclization Cascade for the Biosynthesis of their Bicyclo[4.2.0]octane Core?

Mercedes Cueto, Luis D’Croz, ${ }^{\dagger, \ddagger}$ Juan L. Maté, ${ }^{\dagger} \S$ Aurelio San-Martín ${ }^{\prime}$ and José Darias*

Instituto de Productos Naturales y Agrobiología del CSIC, Avda. Astrofísico F. Sánchez, 3, 38206 La Laguna, Tenerife, Spain

Smithsonian Tropical Research Institute (STRI), P.O. Box 2072, Balboa, Panama

Departamento de Biología Marina y Limnología, Estafeta Universitaria, Universidad de Panamá, Panama

Division of Marine Biology and Fisheries, Rosenstiel School of Marine and Atmospheric Science, University of Miami, 4600 Rickenbacker Causeway, Miami, FL 33149, USA

Dpto de Química, Facultad de Ciencias, Universidad de Chile, Santiago de Chile, Chile

\section{Index:}

Table 1. NMR data of $\mathbf{1}$ and 2

Experiental Section

${ }^{1}$ H NMR spectrum of Elysiapyrone A (1)

${ }^{13} \mathrm{C}$ NMR spectrum of Elysiapyrone A (1)

HSQC spectrum of Elysiapyrone A (1)

HMBC spectrum of Elysiapyrone A (1)

${ }^{1} \mathrm{H}$ NMR spectrum of Elysiapyrone B (2)

${ }^{13} \mathrm{C}$ NMR spectrum of Elysiapyrone B (2)

Table 1. NMR Data of 1 and 2 [500 MHz, $\delta$ ppm, $(J) \mathrm{Hz}$ ]

\begin{tabular}{cccccc}
\hline & \multicolumn{3}{c}{$\mathbf{1}$} & & \multicolumn{2}{c}{$\mathbf{2}$} \\
\cline { 2 - 5 } $\mathrm{n}^{\mathrm{o}}$ & $\delta_{\mathrm{H}}$ & $\delta_{\mathrm{C}}$ & HMBC & $\delta_{\mathrm{H}}$ & $\delta_{\mathrm{C}}$ \\
\hline 1 & --- & 163.2 & & -- & 162.0 \\
2 & --- & 105.4 & & --- & 100.9 \\
3 & --- & 182.5 & & --- & 181.7 \\
4 & --- & 118.9 & & -- & 117.8 \\
5 & --- & 166.1 & & -- & 162.3 \\
6 & --- & 44.9 & & -- & 44.3 \\
7 & $2.27(\mathrm{~s})$ & 54.0 & C-5, C-6, C-8, C-11, & $2.93(\mathrm{~s})$ & 46.9 \\
8 & --- & 59.2 & C-17, C-20 & --- & 56.2 \\
9 & $3.18(\mathrm{~s})$ & 61.1 & C-10, C-11 & $3.08(\mathrm{~s})$ & 60.3 \\
10 & --- & 56.7 & & --- & 56.7 \\
11 & $2.39(\mathrm{~s})$ & 62.1 & C-7, C-12, C-13 & $2.82(\mathrm{~s})$ & 57.8 \\
12 & --- & 35.7 & & --- & 34.0 \\
13 & $2.43 \mathrm{q}(7.5)$ & 43.8 & C-5, C-6, C-11,C-12 & $2.52 \mathrm{q}(6.8)$ & 46.0 \\
14 & $1.19 \mathrm{~d}(7.5)$ & 20.3 & C-6, C-12, C-13 & $1.16 \mathrm{~d}(6.8)$ & 10.0 \\
15 & $1.93(\mathrm{~s})$ & 7.4 & C-1, C-2, C-3 & $1.86(\mathrm{~s})$ & 7.1 \\
16 & $1.89(\mathrm{~s})$ & 11.0 & C-3, C-4, C-5 & $2.08(\mathrm{~s})$ & 10.8 \\
17 & $1.38(\mathrm{~s})$ & 23.4 & C-5, C-6, C-7, C-13 & $1.37(\mathrm{~s})$ & 15.7 \\
18 & $1.55(\mathrm{~s})$ & 22.0 & C-7, C-8, C-9 & $1.40(\mathrm{~s})$ & 21.4 \\
19 & $1.20(\mathrm{~s})$ & 11.2 & C-9, C-10, C-11 & $1.58(\mathrm{~s})$ & 21.0 \\
20 & $1.03(\mathrm{~s})$ & 18.4 & C-7, C-11, C-12, C-13 & $1.17(\mathrm{~s})$ & 26.1 \\
21 & $3.98(\mathrm{~s})$ & 60.4 & C-1 & $4.03(\mathrm{~s})$ & 57.3 \\
\hline
\end{tabular}

† Smithsonian Tropical Research Institute.

\$ Universidad de Panama.

$\$$ University of Miami.

Universidad de Chile 


\section{Experimental}

\section{General procedures}

Optical rotations were measured on a Perkin-Elmer model 343 Plus polarimeter using a $\mathrm{Na}$ lamp at $25{ }^{\circ} \mathrm{C}$. IR spectra were obtained with a Perkin-Elmer 1650/FTIR spectrometer. ${ }^{1} \mathrm{H}$ NMR and ${ }^{13} \mathrm{C}$ NMR, HSQC, HMBC and COSY spectra were measured employing a Bruker AMX 500 instrument operating at $500 \mathrm{MHz}$ for ${ }^{1} \mathrm{H}$ NMR and at $125 \mathrm{MHz}$ for ${ }^{13} \mathrm{C}$ NMR. Two-dimensional NMR spectra were obtained with the standard Bruker software. EIMS and HRMS data were taken on a Micromass Autospec spectrometer. HPLC separations were performed with a Hewlett Packard 1050 (JaigelSil semipreparative column, $10 \mu, 20 \times 250 \mathrm{~mm}$ ) with hexane-EtOAc mixtures. The gel filtration column (Sephadex LH-20) used hexane-MeOH- $\mathrm{CH}_{2} \mathrm{Cl}_{2}(3: 1: 1)$ as solvent. The spray reagent for TLC was $\mathrm{H}_{2} \mathrm{SO}_{4}-\mathrm{H}_{2} \mathrm{O}-\mathrm{AcOH}(1: 4: 20)$.

\section{Biological Material}

One hundred twenty specimens of Elysia diomedea were collected by hand off Saboga Island (Panama) at $-1.5 \mathrm{~m}$.

\section{Extraction and Isolation}

Wet samples were extracted with methanol at room temperature, and were concentrated to give a dark residue $(5.0 \mathrm{~g})$. The extract was partitioned between EtOAc $(3 \times 75 \mathrm{ml})$ and water $(75 \mathrm{ml})$. The EtOAc extracts were combined to obtain a brown oil (643.5 $\mathrm{mg}$ ) that was chromatographed on a LH-20 column. Fractions containing pyrones, as indicated by their ${ }^{1} \mathrm{H}$ NMR spectra, were further chromatographed on HPLC to give compounds $1(0.6 \mathrm{mg})$, and $2(0.6 \mathrm{mg})$ and the known compounds tridachione (77.7 $\mathrm{mg}$ ), 9,10-deoxytridachione (15.2 mg), 15-norphotodeoxytridachione (3.2 $\mathrm{mg}$ ), and iso9,10-deoxytridachione (2.2 $\mathrm{mg})$.

Elysiapyrone A (1). Colorless oil; $[\alpha]_{\mathrm{D}}^{25}=+37^{\circ}\left(c 0.08, \mathrm{CHCl}_{3}\right)$; IR (film): $v_{\max } 1548$ $\mathrm{cm}^{-1}$; EIMS (70 eV): $\mathrm{m} / z(\%): 360\left[\mathrm{M}^{+}\right](6), 345\left[\mathrm{M}^{+}-\mathrm{Me}\right](6), 317\left[\mathrm{M}^{+}-\mathrm{Me}-\mathrm{CO}\right]$ (13), 209 (64), 193 (100); HREIMS 360.1883 (calcd for $\mathrm{C}_{21} \mathrm{H}_{28} \mathrm{O}_{5} 360.1936$ ), 345.1652 (calcd for $\mathrm{C}_{20} \mathrm{H}_{25} \mathrm{O}_{5} 345.17019$ ), 317.1676 (calcd for $\mathrm{C}_{19} \mathrm{H}_{25} \mathrm{O}_{4} 317.1752$ ).

Elysiapyrone B (2). Colorless oil; $[\alpha]_{\mathrm{D}}{ }^{25}=+225^{\circ}$ (c $0.08, \mathrm{CHCl}_{3}$ ); IR (film): $v_{\max } 1548$ $\mathrm{cm}^{-1}$; EIMS (70 eV): m/z (\%) $360\left[\mathrm{M}^{+}\right](20), 345\left[\mathrm{M}^{+}-\mathrm{Me}\right](4), 317\left[\mathrm{M}^{+}-\mathrm{Me}-\mathrm{CO}\right]$ (14), 209 (63), 193 (100); HREIMS 360.1925 (calcd for $\mathrm{C}_{21} \mathrm{H}_{28} \mathrm{O}_{5} 360.1936$ ), 345.1651 (calcd for $\mathrm{C}_{20} \mathrm{H}_{25} \mathrm{O}_{5} 345.17019$ ), 317.1718 (calcd for $\mathrm{C}_{19} \mathrm{H}_{25} \mathrm{O}_{4} 317.1752$ ). 


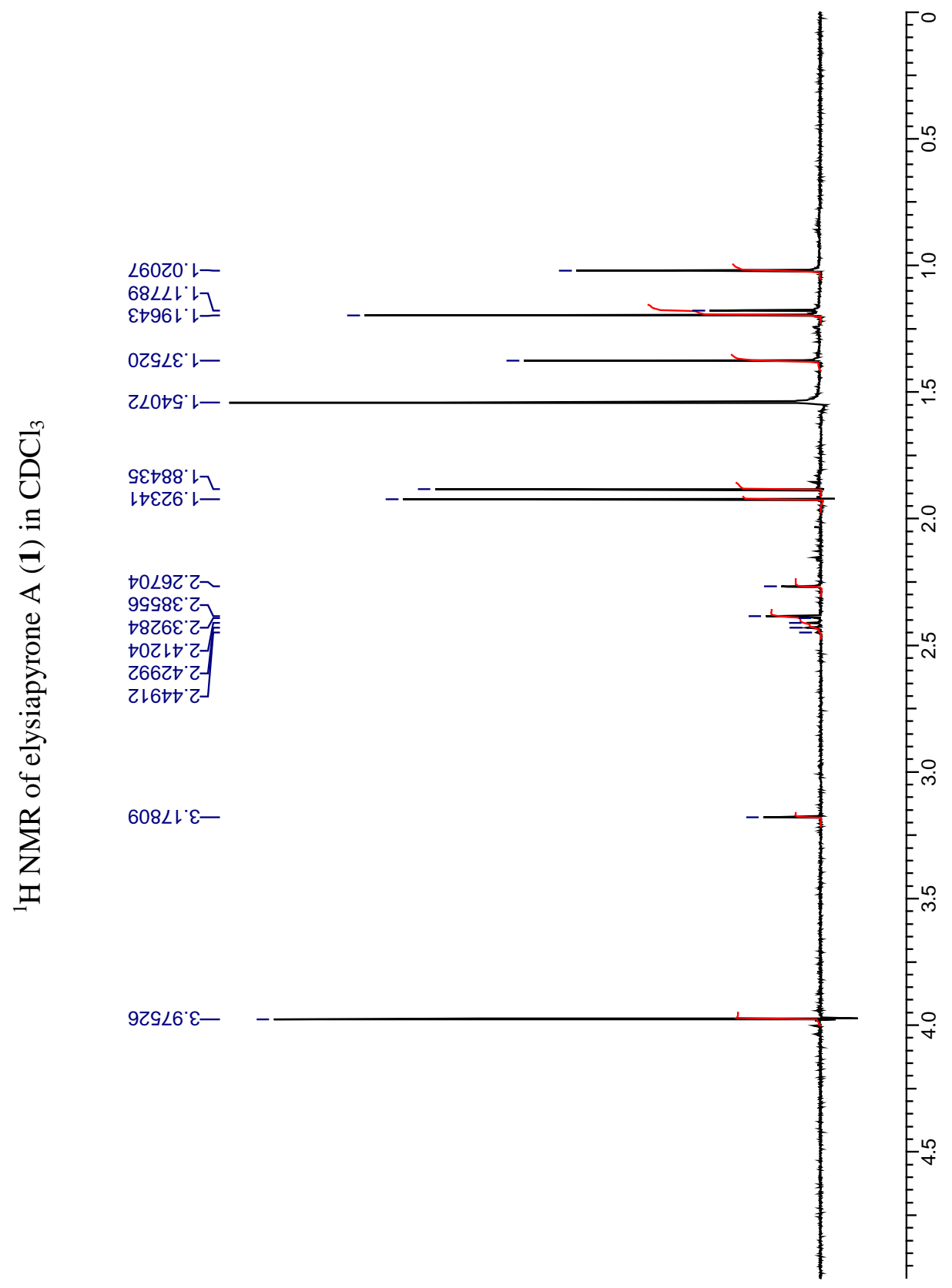




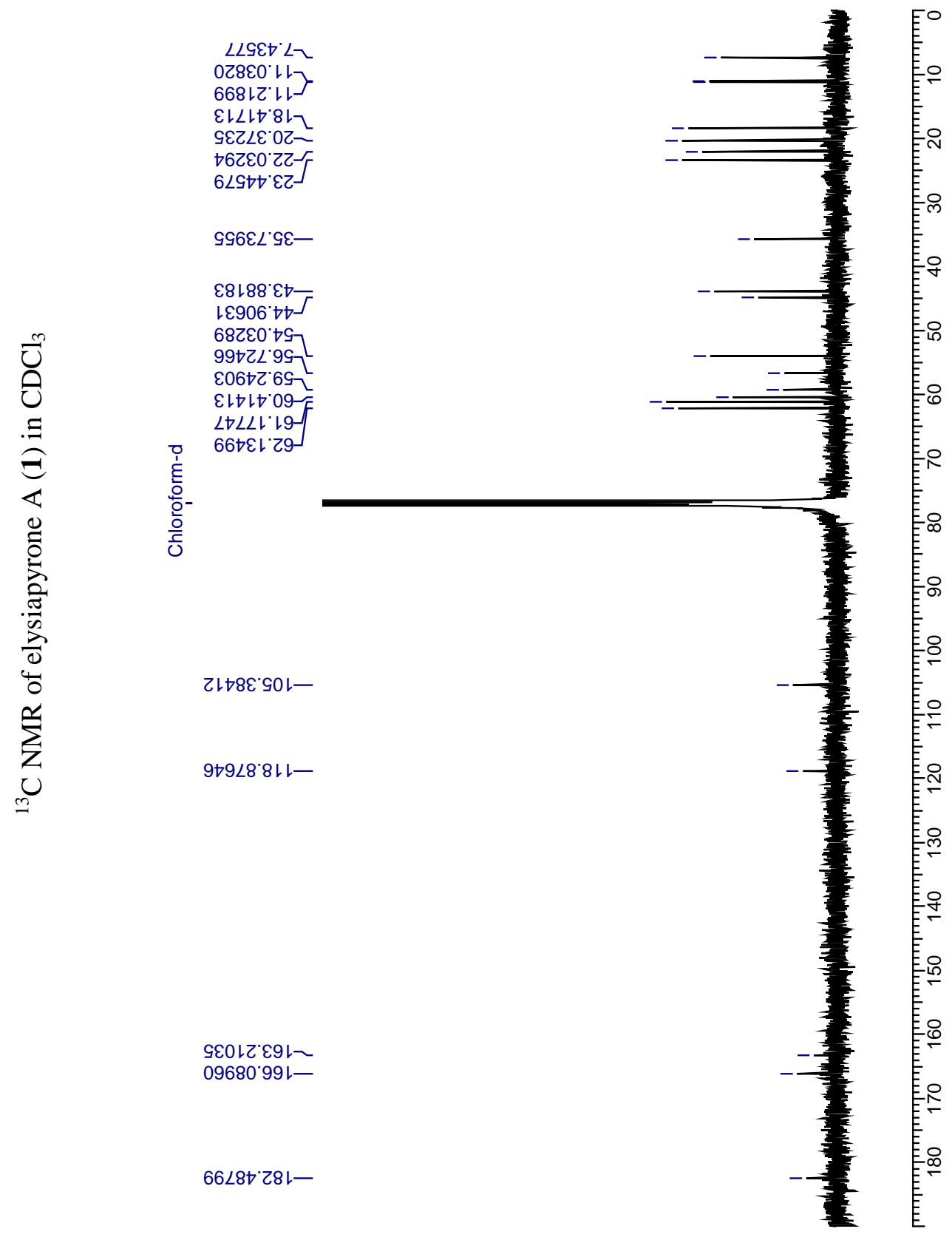




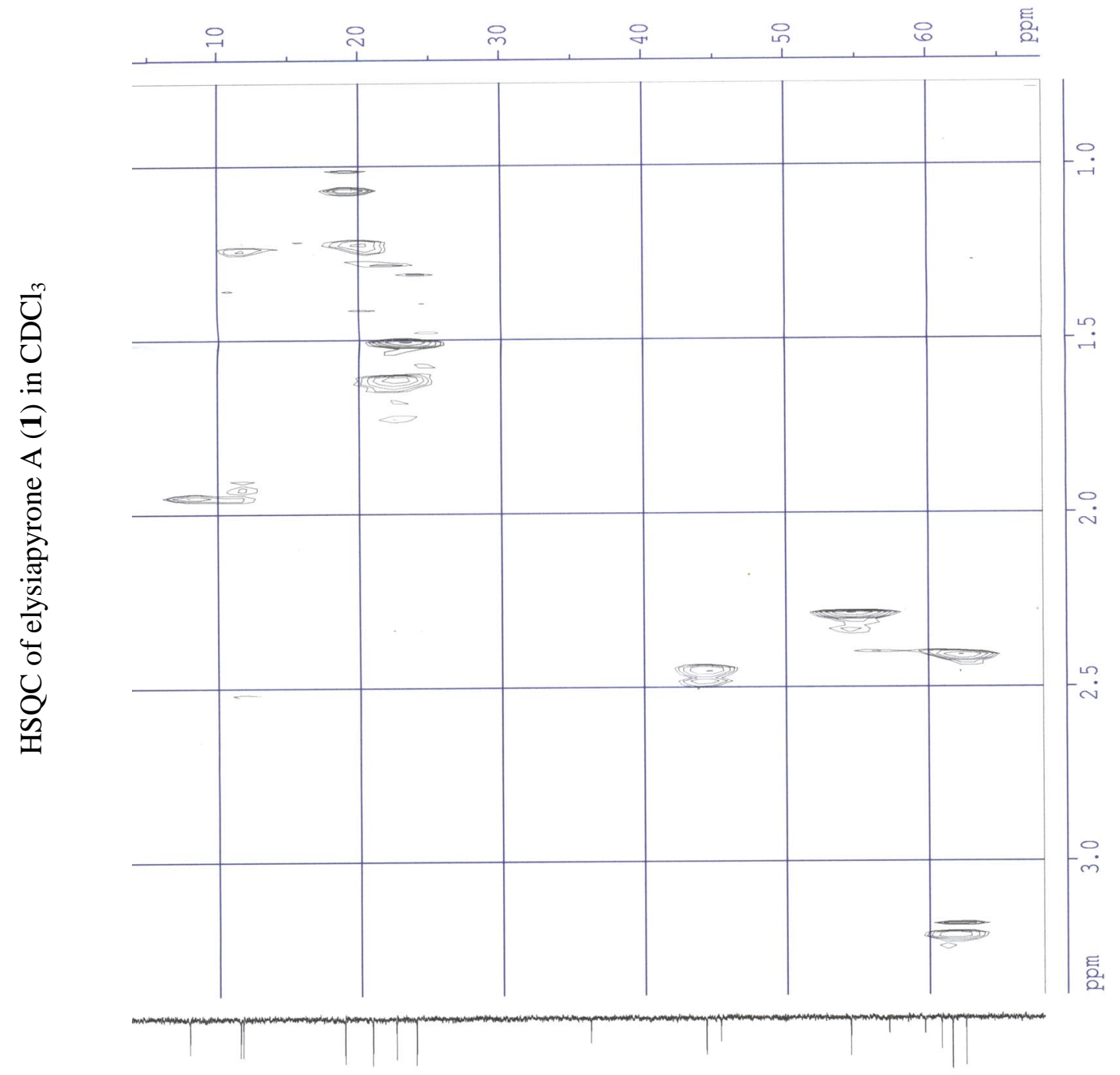




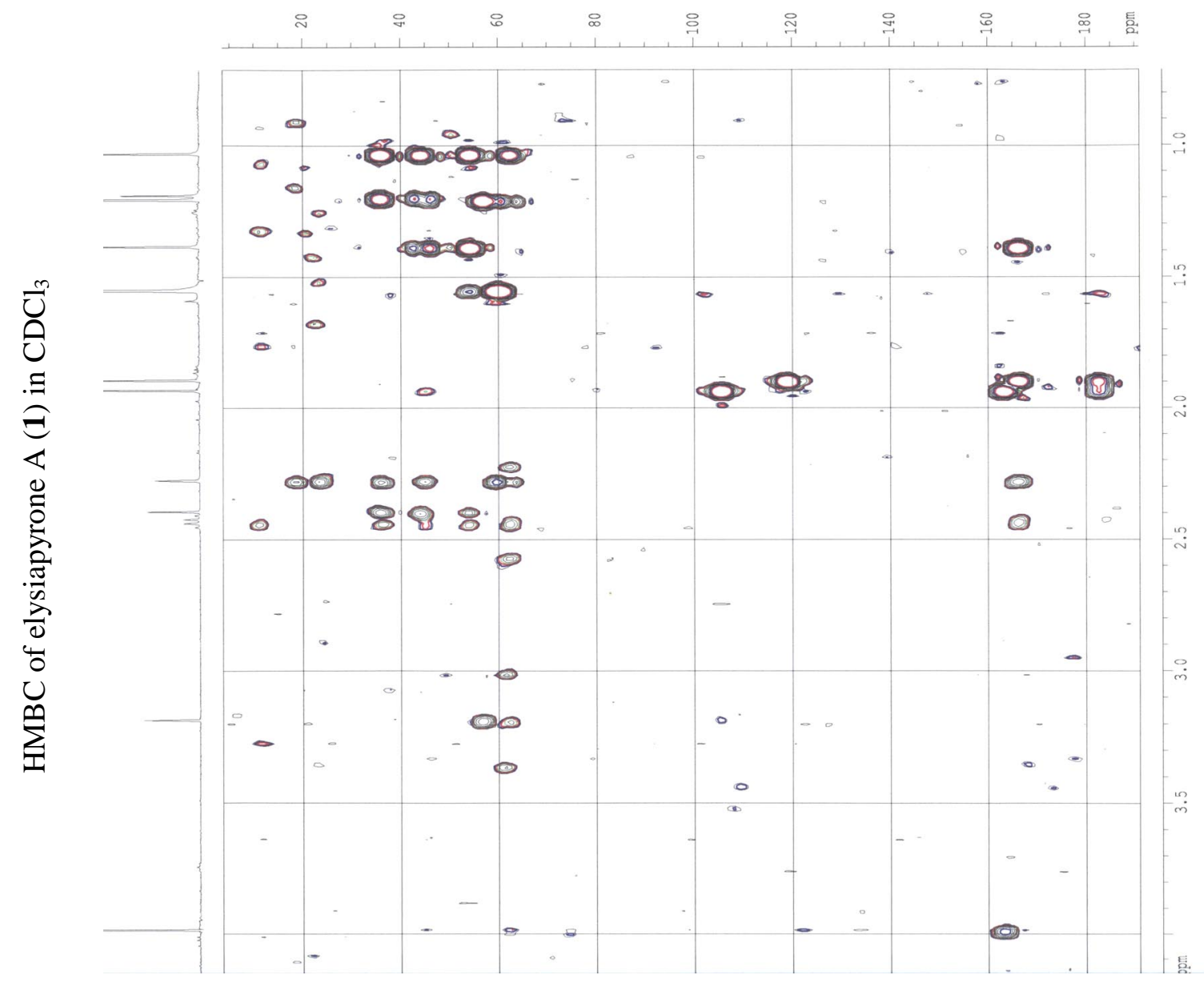




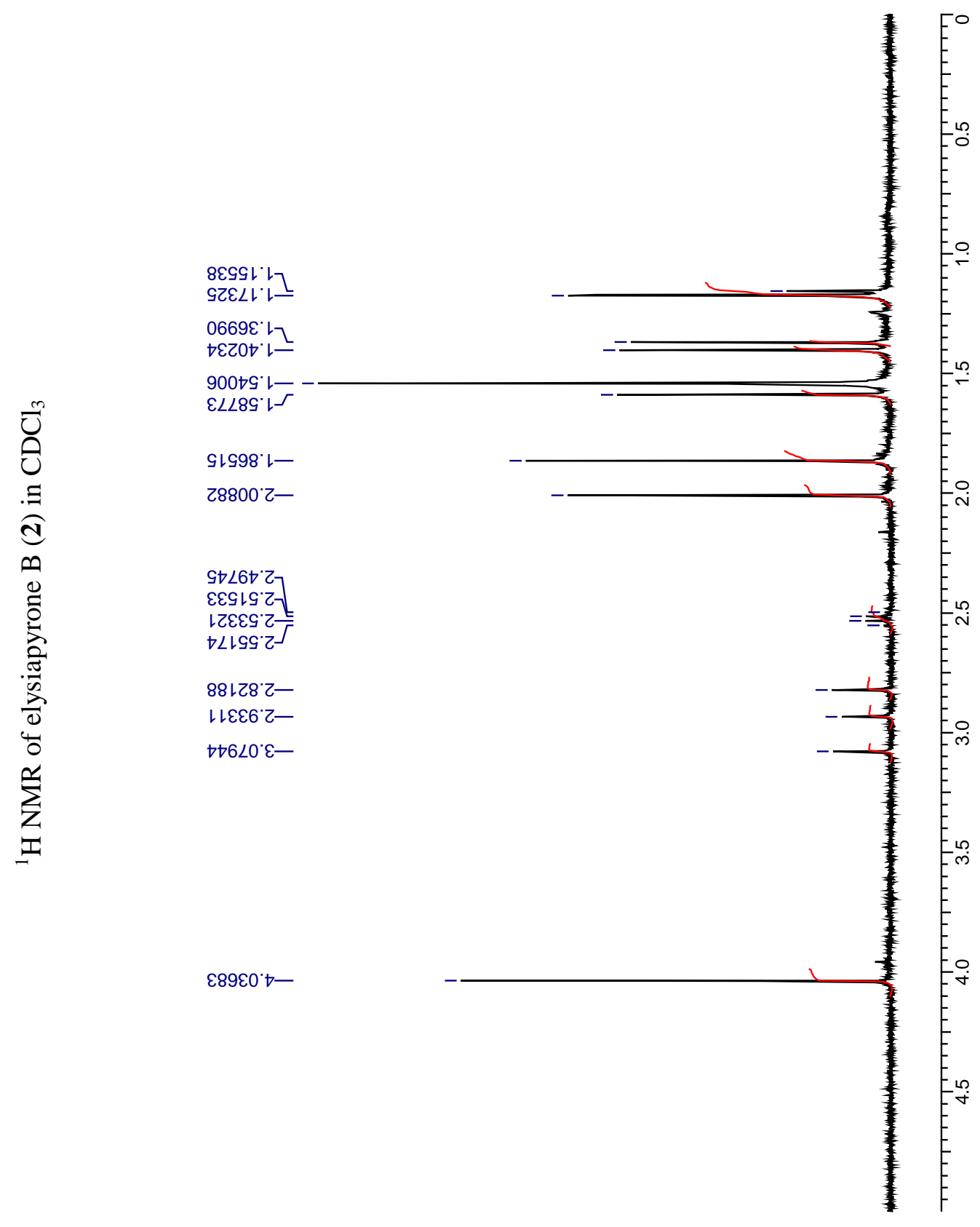




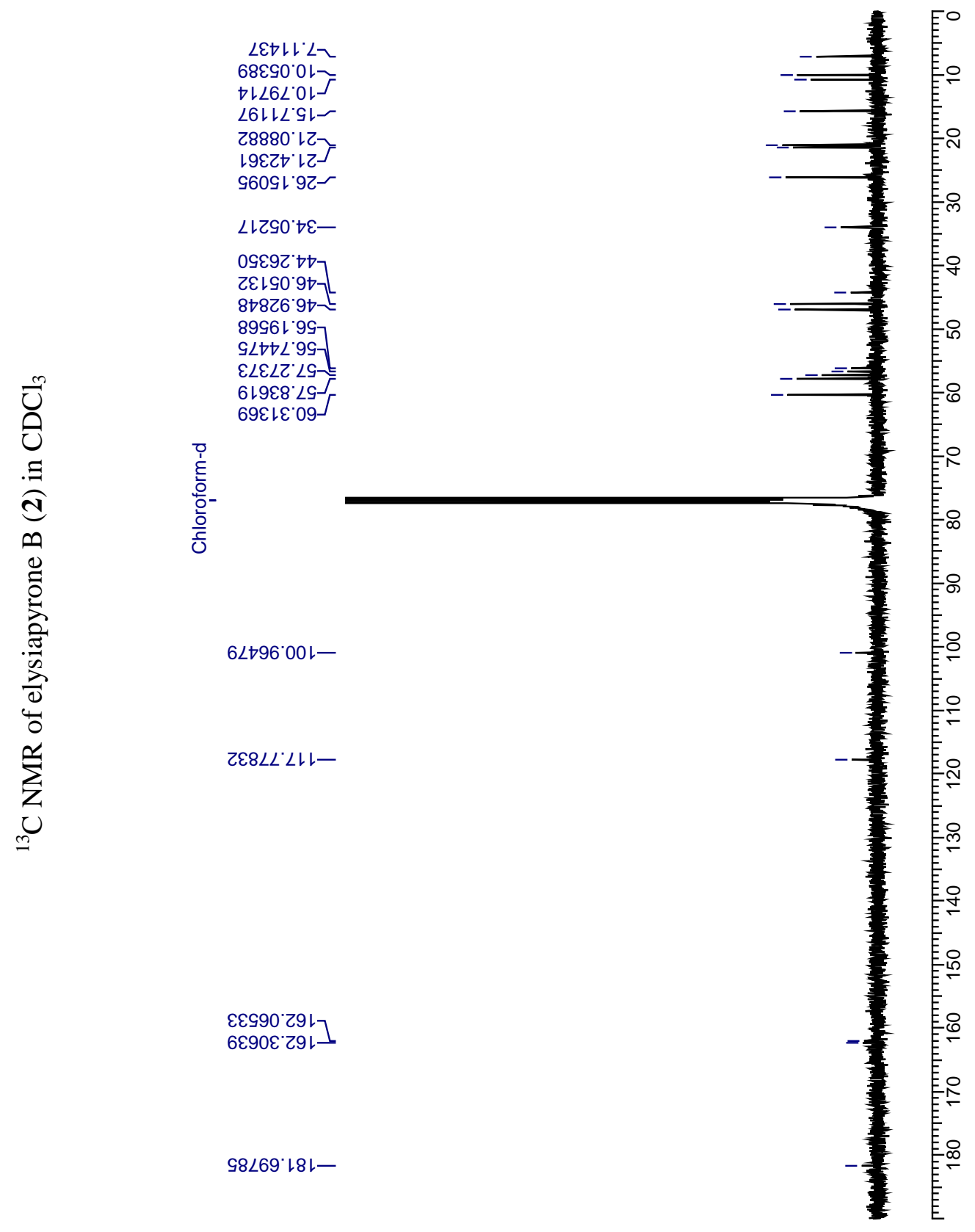

